\title{
Preparation of Nanocarbon-Supported Nanofiber Fabric for Purification of Contaminated Water
}

\author{
Mukai Yasuhito *, Liu Song, Amano Eiji \\ Department of Chemical Systems Engineering, Nagoya University, Furo-cho, Chikusa-ku, Nagoya 464-8603, Japan
}

Received 13 August 2019; accepted for publication 13 December 2019

\begin{abstract}
In this study, a composite fabric was prepared by combining nanofiber having an extremely high specific surface area with nanosized carbon black (CB) having a solute adsorption capacity to purify contaminated water. The nanofiber fabric made of polyacrylonitrile (PAN) with a mean fiber diameter of $400 \mathrm{~nm}$ was used as a base material. The CB particles with an original size of $20 \mathrm{~nm}$ were used as adsorbent and dispersed in pure water at the concentration of $10 \mathrm{~g} / \mathrm{L}$. Before preparation, the $\mathrm{CB}$ particles were oxidized by mixing $\mathrm{CB}$ with $70 \%$ nitric acid at a ratio of $1 \mathrm{~g}$ to $1 \mathrm{~mL}$ and heating for 2 hours with agitation. The surface of $\mathrm{CB}$ particles became hydrophilic by oxidization, and the size of $\mathrm{CB}$ particles became the original one in the dispersion. The nanofiber fabric was immersed in the $\mathrm{CB}$ dispersion and shaken for 2 hours. After taking out and rinsing it lightly, $\mathrm{CB}$ was immobilized on the surface of nanofibers by heating for 1 hour at $150{ }^{\circ} \mathrm{C}$. The adsorption performances of the different $\mathrm{CB}$ particles and the prepared $\mathrm{CB}$-supported PAN nanofiber fabrics were evaluated by the static adsorption test using methylene blue (MB) as an adsorbate. The saturated adsorption amount of the oxidized CB-supported PAN nanofiber fabric was as high as $96.2 \mathrm{mg} / \mathrm{g}$. The dynamic adsorption test of MB was also carried out on the untreated CB-supported PAN nanofiber fabric and the oxidized CB-supported PAN nanofiber fabric. Model equations based on the pseudo-first-kinetic model were derived to analyze the various parameters of the dynamic adsorption. The obtained results suggested a potential application of the oxidized CB-supported PAN nanofiber fabric in the contaminated water treatment field.
\end{abstract}

Key Words : Nanofiber fabric, Carbon black, Composite, Adsorption, Water purification

\section{Introduction}

Organic dyes are widely used in many industries, such as textile finishing, cosmetics, printing, and plastic [1]. Environmental pollution caused by organic dyes in contaminated water has received increased concern in recent years because of the rapid development of urbanization and industrialization [2, 3]. Almost all of these dyes are synthetic and composed of complex aromatic structures [4]. Water contaminated by these dyes poses a tremendous threat to ecological and human health [5]. A variety of physical and chemical strategies have been developed for the removal of the dyes in water, such as chlorination, ozonation, photocatalytic degradation, adsorption, and electrochemical methods [6-9]. Nevertheless, the use of these techniques is limited by high operation cost and ineffectiveness in treating effluent with low dye concentrations. Among these methods, adsorption has been widely used due to its relatively low-cost, efficiency, and ease of operation.
The adsorption process uses an adsorbent, a material that is able to bind the toxic substance or molecule to its surface. Numerous materials have been prepared for use as adsorbents. Recently, electrospun nanofibers are considered to be promising as an alternative to traditional adsorbents due to high porosity and large specific surface area $[10,11]$. Another advantage of electrospun nanofibers over conventional adsorbents is the modifiable nature, as they can be easily designed to obtain the required functional surface adsorption site or specific structure for providing better adsorptive capacity. A lot of researchers have been devoted to developing functional nanofiber fabrics for the removal of dyes from aqueous solutions [12-14]. Shen et al. have prepared a mesoporous alumina nanofiber for the removal of methyl orange dye [15]. The mesoporous alumina nanofiber was obtained after calcining the as-prepared polyvinylpyrrolidone/alumina nanofiber in air above $450{ }^{\circ} \mathrm{C}$ for 6 hours. Zarrini et al. have fabricated a type of nylon6-polyaniline nanocomposite web through coating the surface of nylon-6 nanofibers with polyaniline via in-situ polymerization [16].

* Corresponding author: E-mail : mukai.yasuhito@material.nagoya-u.ac.jp, Tel : +81-52-789-3375, Fax : +81-52-789-5300 
The produced nylon-6-polyaniline nanocomposite web adsorbed up to $370 \mathrm{mg} / \mathrm{g}$ methyl orange. Gopakumar et al. used Meldrum's acid as an esterification agent to modify cellulose nanofiber (CNF) based polyvinylidene fluoride (PVDF) membrane via a nonsolventassisted methodology [2]. Crystal violet dye's adsorption by the PVDF electrospun fiber was $1.368 \mathrm{mg} / \mathrm{g}$, and that of the unmodified CNF-based PVDF fiber was $2.948 \mathrm{mg} / \mathrm{g}$. In contrast, the crystal violet dye's adsorption by the modified CNF-based PVDF fiber was $3.984 \mathrm{mg} / \mathrm{g}$, which showed 2 to 3 times higher adsorption than the PVDF electrospun fiber. Gao et al. calcined the electrospun polyvinyl alcohol (PVA) $-\mathrm{Fe}_{3} \mathrm{O}_{4}$ composite fiber at $600^{\circ} \mathrm{C}$ for 4 hours, and obtained a hollow $\alpha-\mathrm{Fe}_{2} \mathrm{O}_{3}$ nanofiber made of rice-like nanorods, which exhibited a methyl orange adsorption capacity of $80.6 \mathrm{mg} / \mathrm{g}$ [17]. Methylene blue (MB) is commonly used in the wood, cotton and silk industries, whose chief risk regarding its presence in wastewater arises from its harmful effect upon exposure to eyes, as well as possibility of nausea, vomiting and diarrhea. Aluigi et al. have extracted keratin protein from wool by sulphitolysis and transformed keratin powder into nanofiber fabric through the electrospinning process [18]. The MB adsorption capacity of the obtained keratin nanofiber fabric is $170 \mathrm{mg} / \mathrm{g}$. The main concerns of these reported studies are either a long adsorption equilibrium time or complex preparation processes along with costly adsorbent materials. Therefore, high efficiency and low-cost adsorbent material is the foremost need of adsorption technique. Polyacrylonitrile (PAN) has been used to produce nanofibers due to its excellent characteristics and commercial availability, as well as its non-toxic nature [19]. Carbon black (CB) has been demonstrated to be an excellent adsorbent for diverse organic compounds, including dyes [20, 21]. PAN nanofiber fabrics are able to provide a platform for dye adsorption with $\mathrm{CB}$ nanoparticles attributing to their nano-size effect and high specific surface area. Furthermore, the adsorption or filtration process of CB-supported nanofiber fabric can avoid the secondary pollution caused by the generation of a large amount of sludge by using a carbon adsorbent.

The objective of this study was to prepare a composite fabric with an excellent adsorption capacity by combining PAN nanofiber fabric with functionalized $\mathrm{CB}$ particles for the purification of contaminated water. Pretreatment of $\mathrm{CB}$ particles and its influence on particle size were investigated. Surfaces of the CB-supported nanofiber fabrics were characterized by scanning electron microscope. Methylene blue was selected as a model dye pollutant to investigate the adsorption property of the prepared composite nanofiber fabrics. The adsorption capacity was evaluated through the static adsorption test, and the dynamic adsorption behavior was also studied through the dynamic adsorption test and the analysis by the derived model equations.

\section{Preparation of CB-supported nanofiber fabric}

\subsection{Pretreatment of CB particles}

Carbon black (CB) (Nominal diameter $20 \mathrm{~nm}$ ), purchased from Asahi Carbon Co., Ltd. (Japan), was used as an adsorbent. With the aim of improving the adsorption capacity, the $\mathrm{CB}$ particles were subjected to the three following pretreatments: $\mathrm{pH}$ control of $\mathrm{CB}$ dispersion, activation of $\mathrm{CB}$ particles by $\mathrm{KOH}$, and oxidation of $\mathrm{CB}$ particles. (1) $\mathrm{pH}$ control of $\mathrm{CB}$ dispersion: $\mathrm{CB}$ particles were added into pure water at a concentration of $1.0 \mathrm{wt} \%$ and dispersed under ultrasonic irradiation for 20 minutes. Since the initial $\mathrm{pH}$ value of the $\mathrm{CB}$ dispersion was 7.3, $\mathrm{HCl}$ was added to adjust the $\mathrm{pH}$ value to 3.2. (2) Activation of $\mathrm{CB}$ particles by $\mathrm{KOH}$ : The $\mathrm{CB}$ particles were activated by mixing with $\mathrm{KOH}$ and heating for 1 hour at $800{ }^{\circ} \mathrm{C}$ under an atmosphere of $\mathrm{N}_{2}$ gas flow of $0.5 \mathrm{~L} / \mathrm{min}$. Finally, activated $\mathrm{CB}$ particles were rinsed well in water after neutralization with $\mathrm{HCl}$ for the removal of residual $\mathrm{KOH}$. (3) Oxidization of $\mathrm{CB}$ particles: The CB particles were oxidized by mixing with $70 \%$ nitric acid at a ratio of $1 \mathrm{~g}$ to $1 \mathrm{~mL}$ and heating for 2 hours with agitation. Finally, oxidized $\mathrm{CB}$ particles were rinsed well in water for the removal of residual nitric acid.

The particle size distribution of the CB dispersion was measured by a dynamic light scattering device (DLS-7000) manufactured by Otsuka Electronics Co., Ltd. (Japan). Figure 1 shows the size distribution of $\mathrm{CB}$ particles by disparate pretreatments. Comparing with the size distribution of the $\mathrm{CB}$ particles in the dispersions of $\mathrm{pH} 7.3$ and 3.2, the size of the activated CB particles increased, while the size of the oxidized $\mathrm{CB}$ particles reduced as revealed by the distribution data. The calculated average particle size results are displayed in Table 1. The average diameters of CB particles in the dispersions of $\mathrm{pH} 7.3$ and 3.2 are $60.4 \mathrm{~nm}$ and $50.7 \mathrm{~nm}$, with a slight change. The activation of $\mathrm{CB}$ led to an increase in specific surface area of $\mathrm{CB}$ and simultaneously caused the flocculation of $\mathrm{CB}$ particles, resulting in the average diameter to enlarge to 72.4 $\mathrm{nm}$. The CB particles had the smallest average diameter of $23.5 \mathrm{~nm}$

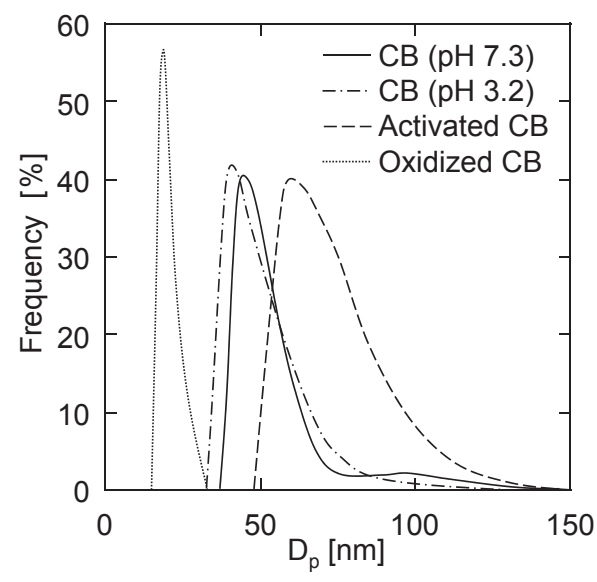

Fig. 1 Size distribution of $\mathrm{CB}$ particles. 
Table 1 Average particle size of CB.

\begin{tabular}{cc}
\hline Pretreatment & $\boldsymbol{D}_{\text {p.av }}[\mathbf{n m}]$ \\
\hline $\mathrm{CB}(\mathrm{pH} 7.3)$ & 60.4 \\
$\mathrm{CB}(\mathrm{pH} \mathrm{3.2)}$ & 50.7 \\
Activated CB & 72.4 \\
Oxidized CB & 23.5 \\
\hline
\end{tabular}

after oxidization, which was close to their original size. Because of the hydrophilic functional groups formed on the CB surface by the oxidation treatment, the oxidized $\mathrm{CB}$ particles were hardly aggregated in water and kept in a well dispersed state.

\subsection{Immobilization of CB onto nanofibers}

The polyacrylonitrile (PAN) nanofiber fabric supplied by Japan Vilene Co. was used as a base material. The mean fiber diameter of the fabric was $400 \mathrm{~nm}$, and the areal weight was $17.2 \mathrm{~g} / \mathrm{m}^{2}$. Four dispersions were prepared by dispersing raw $\mathrm{CB}$ particles at two different $\mathrm{pH}$ environments, activated $\mathrm{CB}$ particles, and oxidized $\mathrm{CB}$ particles separately in pure water. The $\mathrm{pH}$ value of one of the raw $\mathrm{CB}$ dispersions was 7.3 without adjustment and another one was adjusted to $\mathrm{pH}$ 3.2. Round mats of nanofiber fabric with a diameter of $2.5 \mathrm{~cm}$ were immersed in $20 \mathrm{~mL} \mathrm{CB}$ dispersions and shaken for 2 hours. After taking them out from the $\mathrm{CB}$ dispersions and rinsing lightly with pure water, the nanofiber fabrics were dried at $50{ }^{\circ} \mathrm{C}$ for 2 hours. Subsequently, CB particles were immobilized on the surface of nanofibers by heating for 1 hour at $150^{\circ} \mathrm{C}$. In order to remove the unfixed $\mathrm{CB}$ particles, the prepared fibers were immersed into $20 \mathrm{~mL}$ water and shaken for 30 minutes, then washed by an ultrasonic cleaner for 10 minutes. The samples were washed several times until no $\mathrm{CB}$ particles were detected, then again dried at $50{ }^{\circ} \mathrm{C}$ for 2 hours.

Photographs of the original PAN nanofiber fabric mat and the CB-supported nanofiber fabric mats fabricated under various conditions are shown in Fig. 2. The fabric mat mass of original fabric, composite with untreated $\mathrm{CB}$ at $\mathrm{pH} 7.3$, composite with
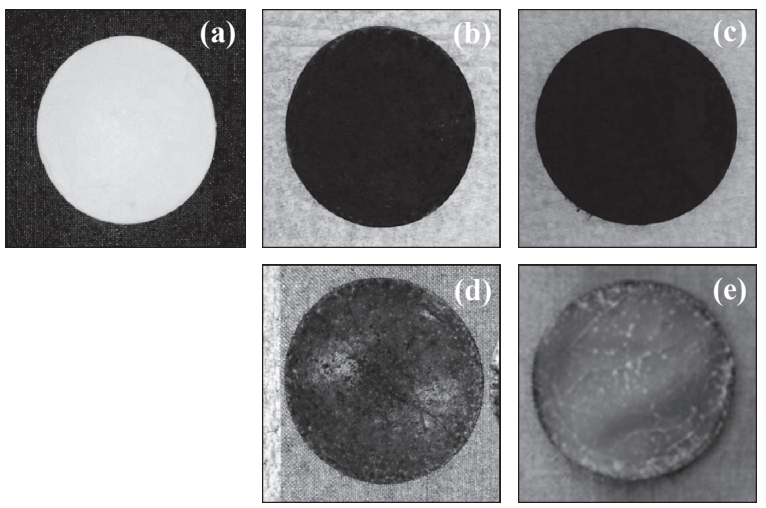

Fig. 2 Appearance of PAN nanofiber fabric mats: (a) original fabric, (b) composite with untreated $\mathrm{CB}$ at $\mathrm{pH} 7.3$, (c) composite with untreated $\mathrm{CB}$ at $\mathrm{pH} \mathrm{3.2,(d)} \mathrm{composite} \mathrm{with} \mathrm{activated}$ $\mathrm{CB}$, (e) composite with oxidized CB.

untreated $\mathrm{CB}$ at $\mathrm{pH} 3.2$, composite with activated $\mathrm{CB}$, and composite with oxidized CB were 8.5, 10.4, 12.1, 9.4, and $10.1 \mathrm{mg}$, respectively. As seen in Fig. 2b and Fig. 2c, both of the untreated CB-supported PAN nanofiber fabric mats exhibited pure black, but the carbon amount immobilized on the fabric under acid condition ( $\mathrm{pH}$ 3.2) is greater. The activated CB-supported PAN nanofiber fabric mat displayed black-gray from the appearance, as presented in Fig. 2d. Activated CB particles were difficult to fix on the surface of the fabric because of a broad particle size distribution caused by flocculation. The CB particles with small size were fixed, while the larger diameter particles were prone to fall off during the rinsing process. In consequence, the amount of $\mathrm{CB}$ particles loading on the fabric reduced and $\mathrm{CB}$ distribution on the fabric surface became uneven, which resulted in the black-gray color of the fabric mat. The oxidized CB-supported PAN nanofiber fabric mat displayed gray, as shown in Fig. 2e. The diameter of CB particles reduced remarkably due to the oxidation treatment, which led to light wavelength reflection inconsistent to that of usual $\mathrm{CB}$ particles. The typical SEM images of original PAN nanofiber fabric mat, composite with untreated $\mathrm{CB}$ at $\mathrm{pH} 7.3$, and composite with oxidized $\mathrm{CB}$ are shown in Fig. 3. The accumulation of $\mathrm{CB}$ particles

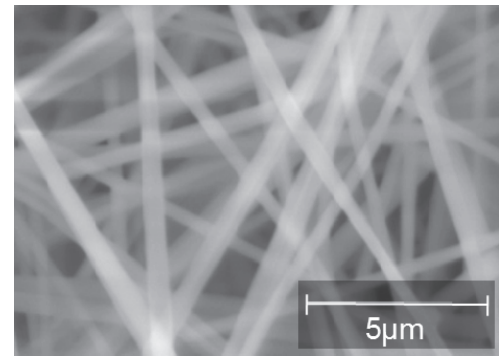

Original fabric

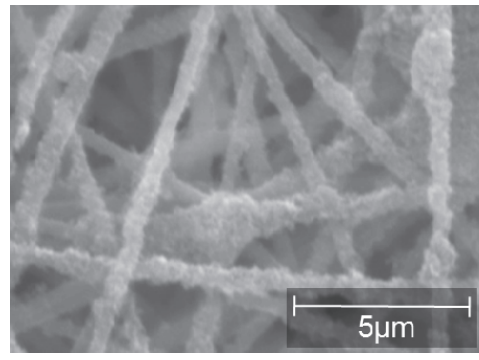

Composite with untreated $\mathrm{CB}$ at $\mathrm{pH} 7.3$

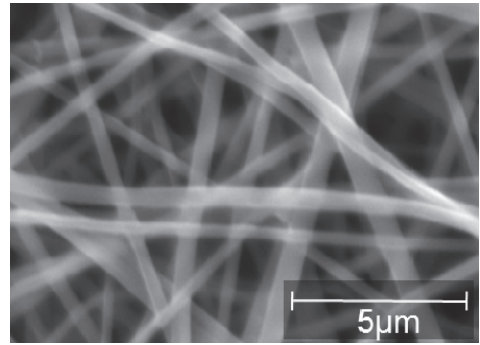

Composite with oxidized CB

Fig. 3 SEM images of PAN nanofiber fabric mats. 
can be observed on the fiber surface of the untreated CB-supported PAN nanofiber fabric, which is corresponded to the pure black color on the photograph of Fig. 2b. Interestingly, the CB particles on the fiber surface of the oxidized CB-supported PAN nanofiber fabric were too small to be observed by SEM. The ultrafine and monodispersed CB particles distributed evenly on the surface of the fiber, showing the gray color in Fig. 2e. In addition, both untreated CBsupported PAN nanofiber fabric, and oxidized CB-supported PAN nanofiber fabric showed a conventional structure without distinct change in morphological structure compared with the original PAN nanofiber fabric.

\section{Adsorption results and discussion}

\subsection{Static adsorption on CB particles}

Methylene blue (MB) $\left(\mathrm{C}_{16} \mathrm{H}_{18} \mathrm{~N}_{3} \mathrm{SCl} \cdot 3\left(\mathrm{H}_{2} \mathrm{O}\right), \mathrm{M}_{\mathrm{w}}=373.90\right)$ was supplied by Wako Pure Chemical Industries, Ltd. (Japan). A standard stock solution of MB dye was prepared by dissolving a certain amount of dye into pure water to get $200 \mathrm{mg} / \mathrm{L}$ of $\mathrm{MB}$ solution. Afterwards, different concentrations were obtained from a series of dilution. To investigate the static adsorption properties, $25 \mathrm{~mL}$ of $\mathrm{CB}$ dispersion was mixed with $25 \mathrm{~mL}$ of $\mathrm{MB}$ aqueous solution at different concentrations, and the mixed solution was stirred at $300 \mathrm{rpm}$ for 1.5 hours. The CB particles were separated from the solution by a centrifugal filter with a pore diameter of 0.1 $\mu \mathrm{m}$ at $3000 \mathrm{rpm}$ for 5 minutes. The centrifugal filter was washed three times by MB filtrate in advance to reduce the error caused by the adsorption of MB on the centrifuge components. The MB concentration was measured by UV-vis spectroscopy (UV-1800, Shimadzu Corp. (Japan)) at a wavelength of $665 \mathrm{~nm}$. The amount of adsorbed MB was calculated based on the following formula:

$$
\Gamma=\frac{\left(C_{0}-C\right) V}{W}
$$

where $\Gamma$ is the adsorption amount $(\mathrm{mg} / \mathrm{g}), C_{0}$ is the initial concentration of MB (mg/L), $C$ is the final concentration of MB $(\mathrm{mg} /$ $\mathrm{L}), V$ is the volume of MB solution (L), and $W$ is the weight of the adsorbent (g).

The effect of initial MB concentration on the adsorption capacity was studied in order to determine the maximum loading capacity of various $\mathrm{CB}$ particles. The isothermal adsorption process of the CB particles fits the Langmuir isothermal adsorption models well. The Langmuir model is based on the assumption of adsorption homogeneity, such as equally available adsorption sites, monolayer surface coverage, and no interaction between adsorbed species. The adsorption process can be expressed by the following equation:

$$
\Gamma=\frac{\Gamma_{\mathrm{s}} K C^{*}}{1+K C^{*}}
$$

where $\Gamma_{\mathrm{s}}$ is the saturated adsorption capacity $(\mathrm{mg} / \mathrm{g}), C^{*}$ is the equilibrium concentration of $\mathrm{MB}$ in solution $(\mathrm{mg} / \mathrm{L})$, and $K$ is the dissociation constant of the system $(\mathrm{L} / \mathrm{mg})$. The linear form of Langmuir equation (2) is depicted as:

$$
\frac{C^{*}}{\Gamma}=\frac{C^{*}}{\Gamma_{\mathrm{s}}}+\frac{1}{K \Gamma_{\mathrm{s}}}
$$

This equation means that the values of $\Gamma_{\mathrm{s}}$ and $K$ are determined from the slope and the intercept of the linear line by plotting $\left(C^{*} / \Gamma\right)$ versus $C^{*}$

The adsorption isotherm $\Gamma$ versus $C^{*}$ in the static adsorption of $\mathrm{MB}$ onto $\mathrm{CB}$ particles is shown in Fig. 4, comparing the experimental data with the calculations according to Eq. (2). The amount of MB adsorbed on the $\mathrm{CB}$ particles increased with the rise in the equilibrium concentration of MB. Significantly, the adsorption capacity of the activated $\mathrm{CB}$ particles and the oxidized $\mathrm{CB}$ particles was higher than that of untreated $\mathrm{CB}$ particles. The saturated adsorption capacity obtained from Eq. (3) is listed in Table 2. The saturated adsorption capacity of the activated CB particles was $359.5 \mathrm{mg} / \mathrm{g}$, which is 7.6 times greater than that of the untreated CB particles, $47.0 \mathrm{mg} / \mathrm{g}$. The BET specific surface area of the activated CB particles was $773.9 \mathrm{~m}^{2} / \mathrm{g}$, which is 7.4 times larger than that of the untreated CB particles, $105.2 \mathrm{~m}^{2} / \mathrm{g}$. This indicates that the increase of specific surface area contributed to the rise of the adsorption quantity. Otowa et al. argued that the metallic potassium was mobile at the activation temperature, and was intercalated to the carbon matrix [22]. As a result, several atomic layers of carbon widened and formed pores. They found that the petroleum coke placed next to the activation zone of $800{ }^{\circ} \mathrm{C}$

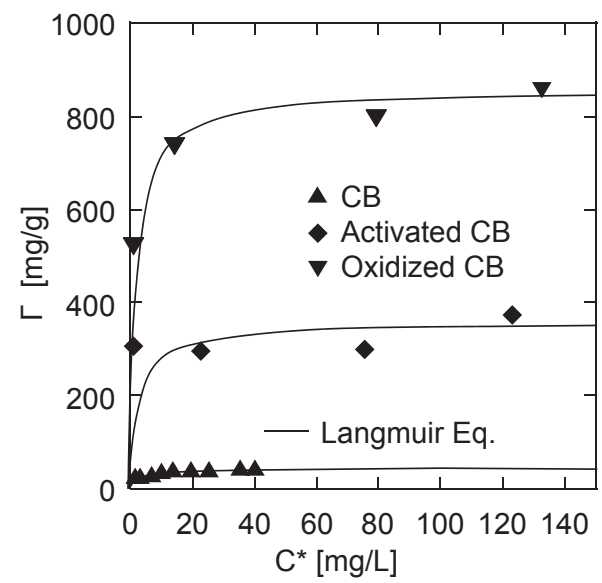

Fig. 4 Adsorption isotherms of MB onto different $\mathrm{CB}$ particles.

Table 2 Saturated adsorption capacity of different CB particles.

\begin{tabular}{cc}
\hline Pretreatment & $\Gamma_{\mathbf{s}}[\mathbf{m g} / \mathbf{g}]$ \\
\hline $\mathrm{CB}$ & 47.0 \\
Activated CB & 359.5 \\
Oxidized CB & 855.4 \\
\hline
\end{tabular}


was activated, and the BET specific surface area was about $800 \mathrm{~m}^{2} /$ g which was very close to $773.9 \mathrm{~m}^{2} / \mathrm{g}$ obtained in our study. After all, the oxidized CB particles had the largest adsorption capacity of $855.4 \mathrm{mg} / \mathrm{g}$. As seen in Table 1, the average diameter of the CB particles dropped from $60.4 \mathrm{~nm}$ to $23.5 \mathrm{~nm}$ by the hydrophilic functional groups formed during the oxidation process, which was $1 / 2.6$ of the untreated CB particles. This indicates that the specific surface area of the oxidized CB particles increased 2.6 times to that of the untreated $\mathrm{CB}$ particles. In contrast, the adsorption capacity of the oxidized $\mathrm{CB}$ particles presented 18.2 times larger than that of the untreated CB particles as seen in Table 2. The decrease of particle diameter was one of influential factors on the adsorption capacity, but affinity created by the functional groups was the main reason for the increase of the adsorption amount. Asai et al. discussed the functional groups such as carboxyl, carbonyl, and phenolic groups formed on the CB surface by nitric acid oxidation [23]. Due to the affinity of these functional groups, the oxidized CB particles exhibited significantly high adsorption performance in our experiments. EI-Hendawy detected the surface-chemical changes of activated carbon by FTIR to invest the impact of oxidation with nitric acid [24]. They observed the absorption bands assigned to carboxyl, carbonyl, and phenolic groups. Also, they emphasized that the surface oxygen groups enhance the hydrophilic character of the carbon surface.

\subsection{Static adsorption on CB-supported nanofiber fabric}

The static adsorption test of the prepared CB-supported nanofiber fabrics was conducted by soaking them into the $\mathrm{MB}$ aqueous solutions at varied concentrations with shaking until a state of adsorption equilibrium was reached. Then the equilibrium concentration $C^{*}$ and the equilibrium adsorption amount $\Gamma$ of MB were measured. The experimental data and the calculations according to Eq. (2) of the adsorption isotherms of MB onto the different fabrics are shown in Fig. 5a. In the static adsorption test of five fabrics, except for the oxidized CB-supported nanofiber fabric,
Table 3 Saturated adsorption capacity of different PAN nanofiber fabrics.

\begin{tabular}{cc}
\hline PAN Fabrics & $\Gamma_{\mathrm{s}}[\mathbf{m g} / \mathbf{g}]$ \\
\hline Without CB & 1.9 \\
With CB (pH 7.3) & 5.0 \\
With CB (pH 3.2) & 6.1 \\
With Activated CB & 18.3 \\
With Oxidized CB & 96.2 \\
\hline
\end{tabular}

the equilibrium adsorption amount of the other fabrics was less than $20 \mathrm{mg} / \mathrm{g}$. An enlargement of Fig. 5a, where the equilibrium adsorption amount was less than $20 \mathrm{mg} / \mathrm{g}$, is displayed in Fig. $5 \mathrm{~b}$, excluding the data of the oxidized CB-supported nanofiber fabric. Furthermore, the saturated adsorption capacity obtained from Eq. (3) is listed in Table 3.

As a result, the value of $\Gamma_{\mathrm{s}}$ was $1.9 \mathrm{mg} / \mathrm{g}$ for the original nanofiber fabric, $5.0 \mathrm{mg} / \mathrm{g}$ for the untreated CB-supported nanofiber fabric, and $6.1 \mathrm{mg} / \mathrm{g}$ for the untreated CB-supported nanofiber fabric prepared under the acid environment. When CB-supported nanofiber fabric was prepared under the acid environment, the amount of supported CB was slightly increased due to the reduction of electrostatic repulsion between $\mathrm{CB}$ particles and PAN fiber. The value of $\Gamma_{\mathrm{s}}$ was $18.3 \mathrm{mg} / \mathrm{g}$ for activated CB-supported nanofiber fabric. The adsorption amount was increased by the activation of $\mathrm{CB}$, whose specific surface area increased from $105.2 \mathrm{~m}^{2} / \mathrm{g}$ to 773.9 $\mathrm{m}^{2} / \mathrm{g}$, as mentioned before. However, the activation induced the flocculation of $\mathrm{CB}$ particles and consequently reduced the rate of increase in the amount of the supported $\mathrm{CB}$, resulting in a smaller adsorption amount than expected. The saturated adsorption capacity of the oxidized CB-supported nanofiber fabric reached $96.2 \mathrm{mg} / \mathrm{g}$. The distinct increase in the amount of adsorption was attributed to the rise in hydrophilic groups and surface area by the process of $\mathrm{CB}$ oxidization. Thus, the oxidization of $\mathrm{CB}$ is an essential pretreatment to attain high solute adsorption performance.

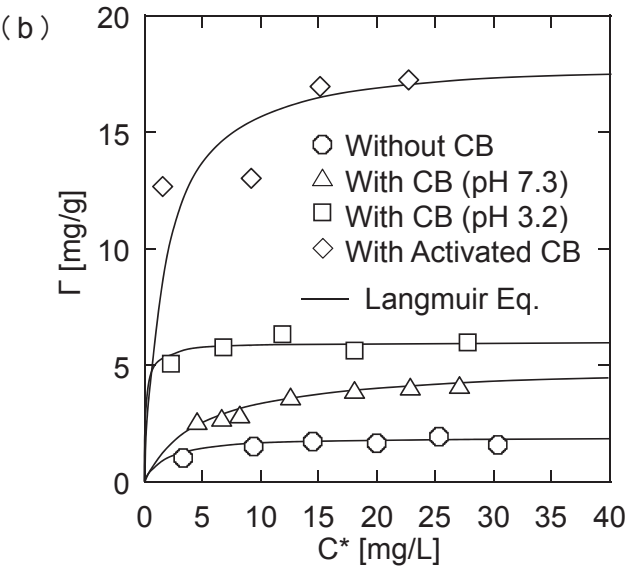

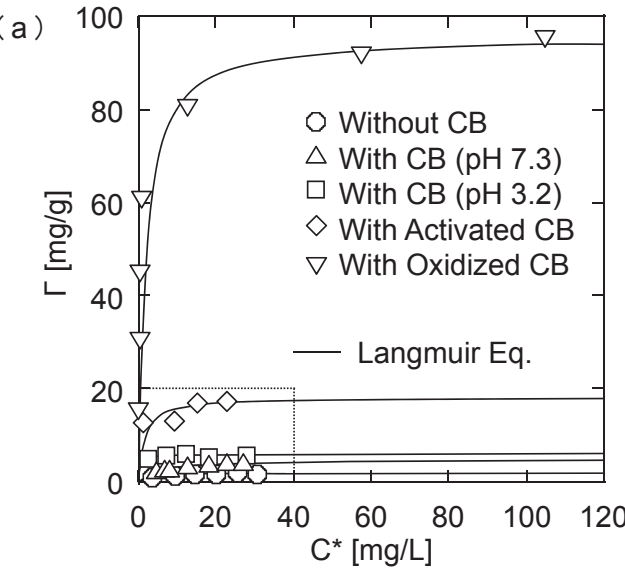

Fig. 5 Adsorption isotherms of MB onto different PAN nanofiber fabrics. 


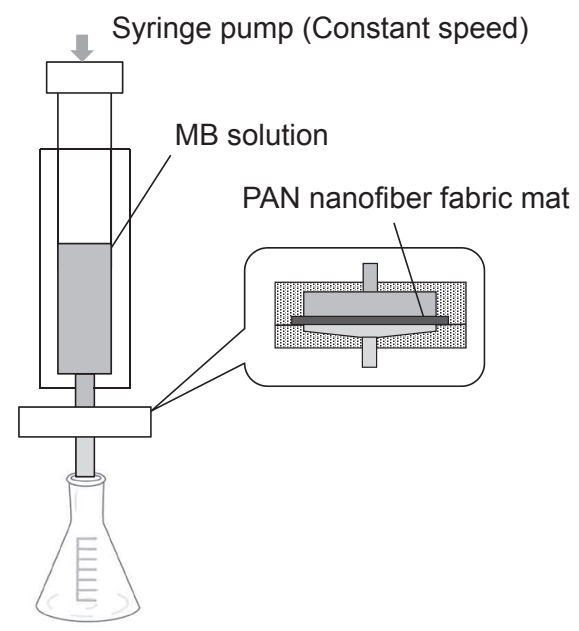

Fig. 6 Experimental set-up of dynamic adsorption.

\subsection{Dynamic adsorption}

\subsubsection{Process of MB dynamic adsorption}

The MB dynamic adsorption test was carried out by permeating the MB solution through the untreated $\mathrm{CB}$ or the oxidized $\mathrm{CB}$ supported PAN nanofiber fabric, as schematically shown in Fig. 6. The fabric mats packed into the filter holder had an effective diameter of $2.2 \mathrm{~cm}$ and a permeation area of $3.8 \mathrm{~cm}^{2}$, and then the filter holder was mounted onto the dynamic adsorption system shown in Fig. 6. Afterwards, MB solution was injected into the filter holder by the syringe pump (SRS-2, AS ONE Co. (Japan)), in the meanwhile, the MB concentration of solution permeating through the fabric was continuously monitored via the UV detector. Dynamic adsorption performance was evaluated under various conditions involving different fabric mat mass $W_{\mathrm{f}}(0.01,0.03$, $0.05 \mathrm{~g}$ ), initial MB concentration $C_{0}$ (from 0.08 to $30 \mathrm{mg} / \mathrm{L}$ ), and permeation rate $q_{\mathrm{v}}$ (from 10 to $50 \mathrm{~mL} / \mathrm{h}$ ).

\subsubsection{Mathematical model for MB dynamic adsorption}

A mathematical model was derived to analyze the results of the dynamic adsorption test. Assuming that adsorption rate, in accordance with the pseudo-first-kinetic model $[25,26]$, is proportional to the adsorption sites number of adsorbent, the following formula can be expressed:

$$
\frac{d \Gamma_{\mathrm{t}}}{d t}=k\left(\Gamma_{\infty}-\Gamma_{\mathrm{t}}\right)
$$

where $\Gamma_{\mathrm{t}}$ is the adsorption amount $(\mathrm{kg} / \mathrm{kg})$ at arbitrary time $t(\mathrm{~s}), \Gamma_{\infty}$ is the adsorption amount $(\mathrm{kg} / \mathrm{kg})$ at $t=\infty, k$ is the rate constant $\left(\mathrm{s}^{-1}\right)$. Integrating Eq. (4), $\Gamma_{\mathrm{t}}$ can be expressed as:

$$
\Gamma_{\mathrm{t}}=\Gamma_{\infty}\{1-\exp (-k t)\}
$$

Rejection $R$ is defined in terms of the fraction of the species in the feed that is retained by the filter medium:

$$
R=1-C_{\mathrm{p}} / C_{0}
$$

where $C_{0}$ is the MB concentration of feed solution $\left(\mathrm{kg} / \mathrm{m}^{3}\right)$, and $C_{\mathrm{p}}$ is the $\mathrm{MB}$ concentration of permeate solution $\left(\mathrm{kg} / \mathrm{m}^{3}\right)$. Rejection can also be defined as the ratio of $\mathrm{MB}$ adsorption rate $W_{\mathrm{f}}\left(d \Gamma_{\mathrm{t}} / d t\right)$ to MB inflow rate $C_{0} q_{v}$, given by:

$$
R=\frac{W_{\mathrm{f}}}{C_{0} q_{\mathrm{v}}} \frac{d \Gamma_{\mathrm{t}}}{d t}
$$

where $W_{\mathrm{f}}$ is the weight of the fabric mat $(\mathrm{kg}), q_{\mathrm{v}}$ is the permeating rate $\left(\mathrm{m}^{3} / \mathrm{s}\right)$. Substituting Eqs. (4) and (5) into Eq. (7), where $t=v / q_{v}$, $v$ is the permeate volume $\left(\mathrm{m}^{3}\right)$, results in the following equation:

$$
R=\frac{W_{\mathrm{f}} k \Gamma_{\infty}}{C_{0} q_{\mathrm{v}}} \exp \left(-\frac{k}{q_{\mathrm{v}}} v\right)
$$

Because the value of rejection cannot be greater than 1 ,

$$
\begin{aligned}
& R=1 \quad \text { at } \quad 0 \leq \mathrm{v} \leq \frac{q_{\mathrm{v}}}{k} \ln \frac{W_{\mathrm{f}} k \Gamma_{\infty}}{C_{0} q_{\mathrm{v}}} \\
& R=\frac{W_{\mathrm{f}} k \Gamma_{\infty}}{C_{0} q_{\mathrm{v}}} \exp \left(-\frac{k}{q_{\mathrm{v}}} v\right) \quad \text { at } \quad v>\frac{q_{\mathrm{v}}}{k} \ln \frac{W_{\mathrm{f}} k \Gamma_{\infty}}{C_{0} q_{\mathrm{v}}}
\end{aligned}
$$

Equations (9) and (10) are obtained for the dynamic adsorption model. According to the two equations, the establishment condition of the dynamic adsorption period of $R=1$ is as follows:

$$
\frac{W_{\mathrm{f}} k \Gamma_{\infty}}{C_{0} q_{\mathrm{v}}}>1
$$

where the left side of Eq. (11) is $R$ at $v=0$ in Eq. (8). Taking the logarithm on both sides of Eq. (8),

$$
-\ln R=\frac{k}{q_{\mathrm{v}}} v-\ln \frac{W_{\mathrm{f}} k \Gamma_{\infty}}{C_{0} q_{\mathrm{v}}}
$$

Therefore, after plotting a linear line with $\ln R$ and $v$, the values of $k$ and $\Gamma_{\infty}$ can be calculated from the slope and the intercept. The fitting curves of the rejection can be obtained by substituting $k$ and $\Gamma_{\infty}$ into Eq. (9) or (10).

\subsubsection{Discussion of dynamic adsorption}

The effects of permeation rate on the dynamic adsorption behavior were studied by varying permeation rate $q_{\mathrm{v}}$ of $\mathrm{MB}$ solution through the untreated CB-supported PAN nanofiber fabric. The experimental data and the calculated rejection based on the dynamic adsorption model equations (9) and (10) are plotted in Fig. 7, where the fitting curves of the rejection are indicated by solid lines. The graph shows that the experimental data followed well with the calculated values. With the increase of permeate volume, the rejection revealed a general trend of decrease though the margins of the decline varied. A noticeable reduction in the rejection occurred 


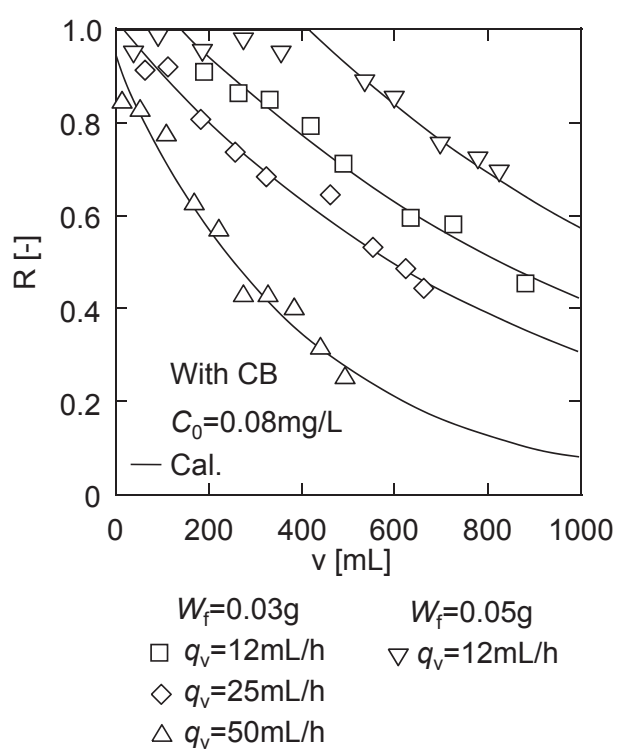

Fig. 7 Dynamic adsorption test results and calculated rejection curves at different permeation rates.

during a permeation rate of $50 \mathrm{~mL} / \mathrm{h}$. For the fabrics of the same mass, there was the same number of adsorption sites for MB capture, while a jump of MB amount caused by a larger permeation rate resulted in the rapid occupation of MB to the fabric surface. In light of the derived Eq. (8), $k \Gamma_{\infty}$ is considered as a critical parameter for evaluating dynamic adsorption performance. Thus, to clarify the correlation between $k \Gamma_{\infty}$ and other parameters in the dynamic adsorption model, the values of $k \Gamma_{\infty}$ calculated from the untreated CB-supported PAN nanofiber fabric are plotted against the permeation rate $q_{\mathrm{v}}$ in Fig. 8 . The graph clearly demonstrates that there was a good linear relation between $k \Gamma_{\infty}$ and the permeation rate. It should be pointed out that $k \Gamma_{\infty}$ had similar values at the fabric mass of $0.03 \mathrm{~g}$ and $0.05 \mathrm{~g}$, when the permeation rate was 12 $\mathrm{mL} / \mathrm{h}$. The results conclusively demonstrated that $k \Gamma_{\infty}$ is related to the permeation rate and is almost unaffected by the mass of the adsorbent.

The influence of the initial solution concentration on the dynamic

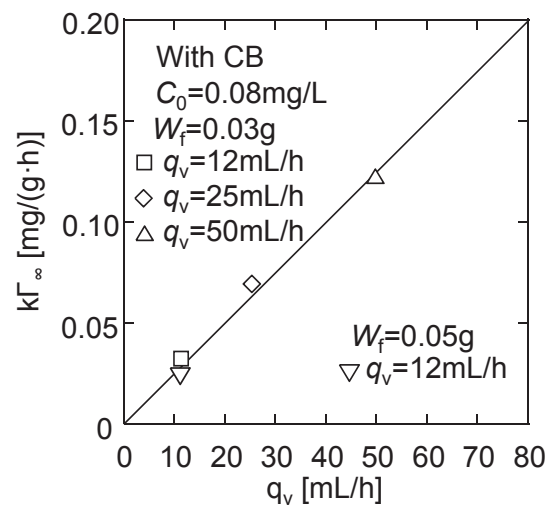

Fig. 8 Correlation between $k \Gamma_{\infty}$ and permeation rate.

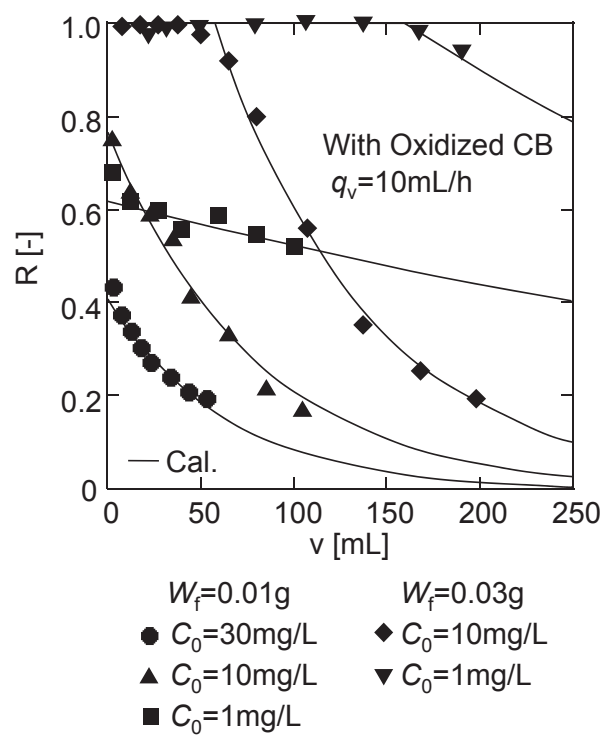

Fig. 9 Dynamic adsorption test results and calculated rejection curves at different initial MB concentrations.

adsorption behavior is an important parameter to investigate since it is altering with the source of effluent and affects the service period of the fabric. Figure 9 shows the dynamic adsorption data at different initial MB concentrations $C_{0}$ for the oxidized CBsupported PAN nanofiber fabric. The rejections calculated according to the dynamic adsorption model equations are plotted by solid lines in Fig. 9 as well. As can be seen, the experimental values fit the calculated values well. The rejection exhibited a sharp descend at an initial MB concentration of $30 \mathrm{mg} / \mathrm{L}$. This is in agreement with the impact of the permeation rate, since a rise of MB amount attributed to the high concentration led to the speedy migration of MB to the fabric surface. In contrast to the rejection values of the adsorption by $0.01 \mathrm{~g}$ fabric, there were dead volume areas in the adsorption by $0.03 \mathrm{~g}$ fabric owing to the abundance of $\mathrm{MB}$ adsorption sites. To explore how the $k \Gamma_{\infty}$ values respond to the initial concentration $C_{0}$, the values of $k \Gamma_{\infty}$ calculated from the oxidized CB-supported PAN nanofiber fabric are plotted against the initial concentration of MB in Fig. 10. In the experimental range, $k \Gamma_{\infty}$ increased roughly

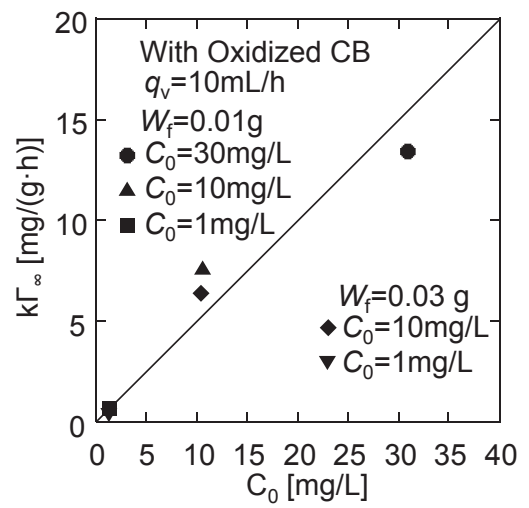

Fig. 10 Correlation between $k \Gamma_{\infty}$ and initial MB concentration. 


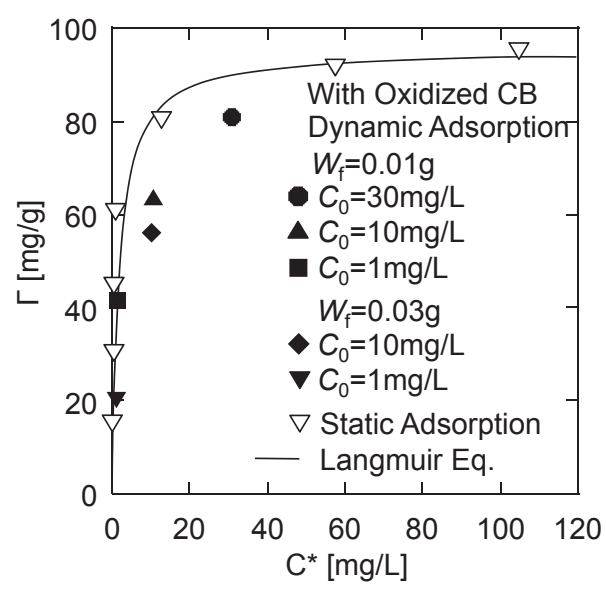

Fig. 11 Comparison between static and dynamic adsorption.

in proportional to $C_{0}$. Notably, $k \Gamma_{\infty}$ had near values at the fabric mass of $0.01 \mathrm{~g}$ and $0.03 \mathrm{~g}$, when the initial concentration was 10 $\mathrm{mg} / \mathrm{L}$. It is safe to say that $k \Gamma_{\infty}$ is an MB amount-related value, independent of the mass of the adsorbent. Accordingly, the dynamic adsorption process in various conditions can be estimated by the model equation after clarifying the permeation rate and the initial concentration.

Additionally, the difference in adsorption capacity between static adsorption and dynamic adsorption was examined in the form of Fig. 11. This figure depicts the adsorption isotherm in static adsorption of the oxidized CB-supported nanofiber fabric, along with the corresponding results of dynamic adsorption. The values of $\Gamma_{\infty}$ obtained after a sufficient adsorption time in dynamic adsorption at varied initial concentrations $C_{0}$ of $\mathrm{MB}$ are assumed to correspond to the values of equilibrium adsorption amount $\Gamma$ at varied equilibrium concentrations $C^{*}$ of $\mathrm{MB}$ in static adsorption. At the corresponding MB concentration, the values of $\Gamma_{\infty}$ were slightly smaller than the equilibrium adsorption amount of $\mathrm{MB}$ in the static adsorption, nevertheless, extremely close to each other. Therefore, by regarding the values of the equilibrium adsorption amount from the static adsorption test as the values of $\Gamma_{\infty}$ and substituting them into the derived dynamic adsorption model equations, the dynamic adsorption process can be estimated approximatively.

\section{Conclusions}

In this research, the composite fabrics were fabricated by immobilizing various $\mathrm{CB}$ particles on the scaffold of PAN nanofiber fabric. The effects of pretreatment on the CB particle size and adsorption properties were investigated. The oxidization process, which increased the CB particles' specific surface area and enhanced the hydrophilicity of the surface, is necessary for $\mathrm{CB}$ before loading on the fabric-based material. The prepared CBsupported PAN nanofiber fabrics were examined for the static and dynamic adsorption behavior of MB. The static adsorption performance of the $\mathrm{CB}$ particles and the CB-supported PAN nanofiber fabrics followed the Langmuir isotherm model. The dynamic adsorption process of the CB-supported nanofiber fabric and the oxidized CB-supported nanofiber fabric was in accordance with the pseudo-first-kinetic model. The adsorption amount $\Gamma_{\infty}$ in the dynamic adsorption calculated by the derived model equations almost coincided with the static adsorption result. Using the oxidized CB-supported nanofiber fabric as an adsorbent material or filtration medium can avoid the secondary pollution caused by traditional materials in contaminated water treatment process. With its high MB adsorption capacity as well as low-cost preparation process, the oxidized CB-supported nanofiber fabric developed in this work might provide a new option for the removal of dyes in contaminated water.

\section{Acknowledgements}

This work was supported in part by JSPS KAKENHI Grant No. 25420801 and No. 16K06824, the Tatematsu Foundation, and Mukai Science and Technology Foundation. The authors would like to express the sincere gratitude for the financial support.

\section{References}

[1] Sham AYW, Notley SM (2018) Journal of Environmental Chemical Engineering, 6, 495-504. https://doi.org/10.1016/ j.jece.2017.12.028

[2] Gopakumar DA, Pasquini D, Henrique MA, Morais LC, Grohens Y, Thomas S (2017) ACS Sustainable Chemistry \& Engineering, 5, 2026-2033. https://doi.org/10.1021/ acssuschemeng.6b02952

[3] Salleh MAM, Mahmoud DK, Karim WAWA, Idris A (2011) Desalination, 280, 1-13. https://doi.org/10.1016/ j.desal.2011.07.019

[4] Hayat H, Mahmood Q, Pervez A, Bhatti ZA, Baig SA (2015) Separation and Purification Technology, 154, 149-153. https:// doi.org/10.1016/j.seppur.2015.09.025

[5] Nasrullah A, Bhat AH, Naeem A, Isa MH, Danish M (2018) International Journal of Biological Macromolecules, 107, 1792-1799. https://doi.org/10.1016/j.ijbiomac.2017.10.045

[6] Gunasekaran R, Kanmani S (2014) Clean Technologies and Environmental Policy, 16, 601-607. https://doi.org/10.1007/ s10098-013-0656-9

[7] Tehrani-Bagha AR, Mahmoodi NM, Menger FM (2010) Desalination, 260, 34-38. https://doi.org/10.1016/ j.desal.2010.05.004

[8] Natarajan S, Bajaj HC, Tayade RJ (2018) Journal of Environmental Sciences, 65, 201-222. https://doi.org/10.1016/ j.jes.2017.03.011

[9] Nidheesh PV, Zhou M, Oturan MA (2018) Chemosphere, 197, 210-227. https://doi.org/10.1016/j.chemosphere.2017.12.195

[10] Hongu T (2016) "Nanofiber Technology", pp42-45, The Nikkan Kogyo Shimbun, Tokyo (in Japanese)

[11] Mukai Y (2011) Nanofiber, 2, 20-25 (in Japanese) 
[12] Yoon K, Hsiao BS, Chu B (2008) Journal of Materials Chemistry, 18, 5326-5334. https://doi.org/10.1039/B804128H

[13] Teng M, Li F, Zhang B, Taha AA (2011) Colloids and Surfaces A: Physicochemical and Engineering Aspects, 385, 229-234. https://doi.org/10.1016/j.colsurfa.2011.06.020

[14] Neghlani PK, Rafizadeh M, Taromi FA (2011) Journal of Hazardous Materials, 186, 182-189. https://doi.org/10.1016/ j.jhazmat.2010.10.121

[15] Shen J, Li Z, Wu Y, Zhang B, Li F (2015) Chemical Engineering Journal, 264, 48-55. https://doi.org/10.1016/j.cej.2014.11.069

[16] Zarrini K, Rahimi AA, Alihosseini F, Fashandi H (2017) Journal of Cleaner Production, 142, 3645-3654. https://doi. org/10.1016/j.jclepro.2016.10.103

[17] Gao Q, Luo J, Wang X, Gao C, Ge M (2015) Nanoscale Research Letters, 10, 176. https://doi.org/10.1186/s11671-015-0874-7

[18] Aluigi A, Rombaldoni F, Tonetti C, Jannoke L (2014) Journal of Hazardous Materials, 268, 156-165. https://doi.org/10.1016/ j.jhazmat.2014.01.012

[19] Almasian A, Fard GC, Gashti MP, Mirjalili M, Shourijeh ZM (2016) Desalination and Water Treatment, 57, 10333-10348. https://doi.org/10.1080/19443994.2015.1041161

[20] Daifullah AAM, Girgis BS, Gad HMH (2004) Colloids and Surfaces A: Physicochemical and Engineering Aspects, 235, 1-10. https://doi.org/10.1016/j.colsurfa.2003.12.020

[21] Karagöz S, Tay T, Ucar S, Erdem M (2008) Bioresource Technology, 99, 6214-6222. https://doi.org/10.1016/ j.biortech.2007.12.019

[22] Otowa T, Tanibata R, Itoh M (1993) Gas Separation \& Purification, 7, 241-245. https://doi.org/10.1016/09504214(93)80024-Q

[23] Asai S, Sakata K, Sumita M, Miyasaka K, Sawatari A (1991) Nippon Kagaku Kaishi, 1991, 1672-1676 (in Japanese). https:// doi.org/10.1246/nikkashi.1991.1672

[24] EI-Hendawy ANA (2003) Carbon, 41, 713-722. https://doi. org/10.1016/S0008-6223(03)00029-0

[25] Lagergren S (1898) Kungliga Svenska Vetenskapsakademiens. Handlingar, 24, 1-39

[26] Simonin JP (2016) Chemical Engineering Journal, 300, 254263. https://doi.org/10.1016/j.cej.2016.04.079 